\title{
Efficacy of perceptual vision therapy in enhancing visual acuity and contrast sensitivity function in adult hypermetropic anisometropic amblyopia
}

This article was published in the following Dove Press journal:

Clinical Ophthalmology

II December 2013

Number of times this article has been viewed

\section{Elvan Yalcin}

Ozlem Balci

World Eye Hospital, Department of Pediatric Ophthalmology, Istanbul, Turkey
Correspondence: Elvan Yalıın

World Eye Hospital, Alemdağ C

Aquamanors 43-AY, Dudullu İstanbul

34000 , Istanbul, Turkey

Tel +905322625719

Fax +90 21 66444083

Email elvanyalcın@hotmail.com
Background: The purpose of this study was to evaluate the efficacy of neural vision therapy, also termed perceptual vision therapy, in enhancing best corrected visual acuity (BCVA) and contrast sensitivity function in amblyopic patients.

Methods: This prospective study enrolled 99 subjects previously diagnosed with unilateral hypermetropic amblyopia aged 9-50 years. The subjects were divided into two groups, with 53 subjects (53 eyes) in the perceptual vision therapy group and 46 subjects (46 eyes) in the control group. Because the nature of the treatment demands hard work and strict compliance, we enrolled the minimal number of subjects required to achieve statistically significant results. Informed consent was obtained from all subjects. Study phases included a baseline screening, a series of 45 training sessions with perceptual vision therapy, and an end-of-treatment examination. BCVA and contrast sensitivity function at 1.5, 3, 6, 12, and 18 cycles per degree spatial frequencies were obtained for statistical analysis in both groups. All subjects had followup examinations within 4-8 months. With the exception of one subject from the study group and two subjects from the control group, all subjects had occlusion during childhood. The study was not masked.

Results: The results for the study group demonstrated a mean improvement of 2.6 logarithm of the minimum angle of resolution ( $\log$ MAR) lines in visual acuity (from 0.42 to 0.16 $\log$ MAR). Contrast sensitivity function improved at $1.5,3,6,12$, and 18 cycles per degree spatial frequencies. The control group did not show any significant change in visual acuity or contrast sensitivity function. None of the treated eyes showed a drop in visual acuity. Manifest refractions remained unchanged during the study.

Conclusion: The results of our study demonstrate the efficacy of perceptual vision therapy in improving visual acuity. The $2.6 \log$ MAR lines improvement in visual acuity is encouraging, and is consistent with the results of previous studies. However, long-term follow-up and further studies are needed.

Keywords: perceptual learning, visual acuity, contrast sensitivity function, amblyopia

\section{Introduction}

Amblyopia is the most frequent cause of reduced vision (unilateral or bilateral) in children, and is demonstrated by deficiencies such as loss of Snellen and grating acuity, ${ }^{1,2}$ loss of contrast sensitivity, ${ }^{3}$ and creation of distortions in the perceived shape of a stimulus. ${ }^{4}$ Amblyopia has been estimated to affect $1 \%-5 \%$ of the population, ${ }^{5}$ and may be associated with strabismus, anisometropia, and disruption of normal development of the lateral geniculate body during the neonatal period. This has been well established by quantitative histologic studies in several animal species and in humans. ${ }^{6-11}$ 
However, the initial neural site corresponding to the visual deficit observed in this condition is still under investigation.

The loss of vision is thought to be secondary to abnormal relationships within the neuronal network in the primary visual cortex. Traditional treatment for amblyopia is based on depriving the good eye while optimizing the visual function of the amblyopic eye in childhood. The phrase "perceptual learning" describes a process whereby practicing certain visual tasks leads to improvement in visual performance. Visual performance can be improved with repetitive practice of specific controlled visual tasks. These repetitive tasks initiate neural modifications that can lead to improvement in neuronal efficiency. Perceptual learning is an alternative treatment option which modifies visual function in adult amblyopia. This has been demonstrated by improvement in vernier acuity in adults with amblyopia. ${ }^{12-16}$ Vernier acuity is the ability to align two line segments. Humans can do this with remarkable accuracy.

The perceptual learning therapy program (RevitalVision LLC, Lawrence, KS, USA) is a noninvasive, software-based, patient-specific, interactive perceptual learning tool based on visual stimulation. It facilitates neural connections at the cortical level through a computerized visual training regimen using Gabor patches to improve contrast sensitivity and visual acuity. ${ }^{14,16-19}$ This treatment approach aims to reduce the noise activity of single cells, thereby increasing the signal strength and readapting the cortex to higher contrast sensitivity and visual acuity levels. In the first stage, the patient is exposed to a set of visual perception tasks aiming to analyze and identify neural efficiencies or deficiencies. The basis of this visual stimulation is the Gabor patch (Figure 1), which matches the shape of the receptive field in the visual cortex and activates it

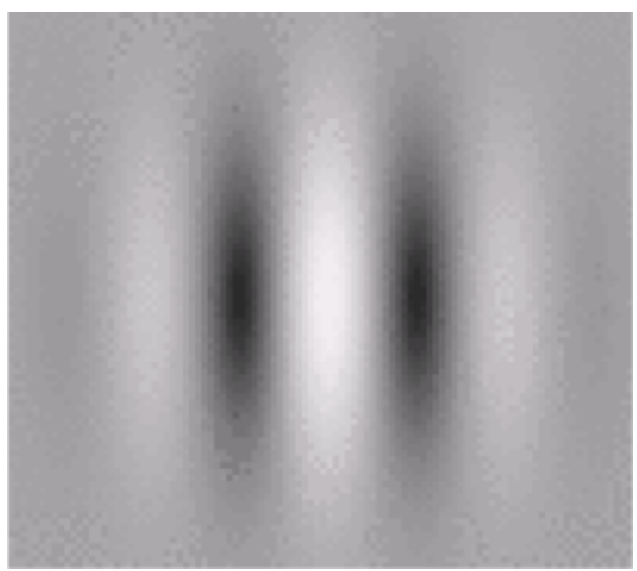

Figure I The Gabor patch. efficiently. The fundamental stimulation-control technique is called "lateral masking" (Figure 2), where collinearly oriented flanking Gabor patches are displayed in addition to the target Gabor image. The patient is exposed to two short displays in a randomized successive manner, after which the patient identifies the display that contained the target Gabor image. An incorrect response by the patient is followed by reinforcing audio feedback. The task is repeated, and a staircase approach is applied until the patient reaches their visual threshold level. Based on this analysis, a patient-specific treatment plan is initialized. Subject specificity is established by administering patient-specific stimuli in a controlled environment.

This study attempted to evaluate the efficacy of perceptual vision (neural vision) therapy in enhancing best-corrected visual acuity (BCVA) and contrast sensitivity function (CSF) in amblyopic patients.

\section{Materials and methods}

This prospective study enrolled 99 amblyopic subjects aged 9-50 years. The patients were divided into two groups, ie, 53 subjects (53 eyes) in a perceptual vision therapy group and 46 subjects (46 eyes) in a control group. Informed consent was obtained from all subjects. The subjects included outpatients who met the inclusion criterion of hypermetropic amblyopia with no history of intraocular surgery, neurologic disease, retinal disease, glaucoma, nystagmus, or strabismus. An amblyopic eye was defined as one with a visual acuity of at least two lines worse than the best-corrected vision of the normal eye using a Snellen chart. Study phases included a baseline screening, a series of 45 training sessions with perceptual vision therapy, and end-of-treatment examination.

All subjects were screened using a detailed baseline eye examination which included manifest refraction, BCVA

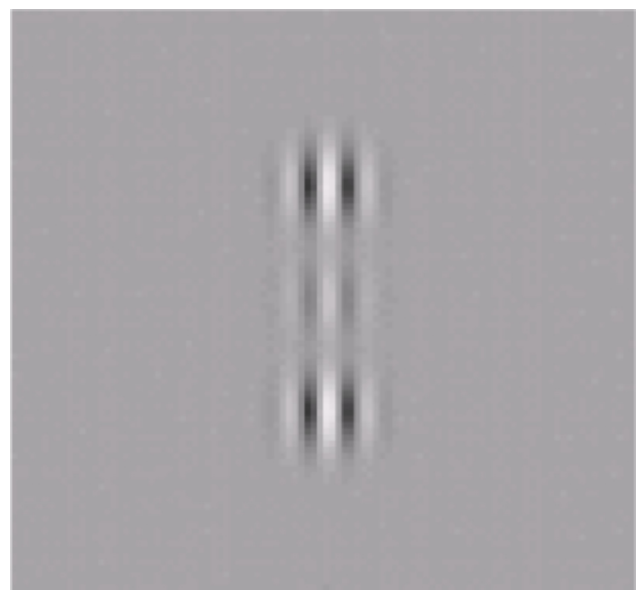

Figure 2 Lateral masking technique. 
using Early Treatment Diabetic Retinopathy Study logMAR chart, cycloplegic refraction using 1\% tropicamide, a cover-uncover test, prism test, extraocular movements, Worth 4 dot test, random dot stereopsis, CSF at 1.5, 3, 6, 12, and 18 cycles per degree spatial frequencies (Optec functional vision analyzer, Stereo Optical Company Inc., Chicago, IL, USA), intraocular pressure measurement, slitlamp biomicroscopy, and fundus examination. Intraocular pressure, anterior segment, and fundus examination were normal in all eyes. All patients from the two groups were best-corrected one month prior to starting therapy. With the exception of one subject from the study group and two subjects from the control group, all subjects had occlusion during childhood. Amblyopic subjects in the study group underwent perceptual vision therapy training. Each training session lasted for approximately 30 minutes during which the subject responded to visual perception tasks displayed on a computer screen. The subjects responded to the tasks using the computer mouse and sitting 5 feet away from the screen. Each session was designed to train those functions in the visual cortex targeted for further enhancement.

At each session, an algorithm analyzed the subject's responses and accordingly adjusted the level of visual difficulty to the range most effective for further improvement. Between sessions, the progress of the subject was used as a baseline for the next session. Thus, for each subject, an individual training schedule was designed based on the initial state of visual performance, severity of dysfunction, and progress in the course of treatment. The treatment was applied in successive 30-minute sessions, administered three times a week, for a total of approximately 45 sessions. Visual acuity and contrast sensitivity were checked at 15-session intervals. The first sessions were performed at the clinic under supervision, with additional sessions performed at home. Each treatment station (home PC) was connected to the central database server via the Internet. After each training session, the results were automatically sent to the server via the Internet. The algorithm, which was located in the central server, calculated specific patient results and sent these back to the specific station as a tailored training task. This unique mechanism enables complete and comprehensive control of each patient performance, including data on exact date and time of training, length of training, and quality of training in high resolution. After completing the 45 training sessions, the subjects were scheduled for an end-of-treatment examination, which included the same basic assessments as the baseline examination. In the study group, there were a total of four visits, ie, a baseline visit, a 15 th session visit (visit 1), a 30th session visit (visit 2), and an end of treatment examination (session 45 visit).

The 46 amblyopic subjects in the control group underwent 30 minutes of eye patching three times a week. They did not perform perceptual vision therapy, and instead played placebo computer games at home. The subjects were followed up bimonthly. At each visit, BCVA and CSF were checked. After completing their placebo computer treatment, the controls were scheduled for an end-of-treatment examination.

All subjects were followed up to 4 months. In the study group, the baseline, 15 th session visit (visit 1), 30th session visit (visit 2), and end-of-treatment data (session 45 visit) were used for the statistical analysis. Compliance was verified automatically by the perceptual vision treatment system. In the control group, the baseline and end-of-treatment data (month 4) were used for statistical analysis. Statistical Package for the Social Sciences version 15 software (SPSS Inc., Chicago, IL, USA) was used for the data analysis along with one-way analysis of variance. A $P$ value less than 0.05 was considered to be statistically significant.

\section{Results}

The perceptual vision therapy group included 53 subjects ( $n=53$ eyes) and the control group included 46 subjects ( $n=46$ eyes), all with unilateral hypermetropic anisometropic amblyopia.

\section{Demographics}

The study group included 30 females and 23 males aged 9-50 (mean 20.3 \pm 8.5 ) years with a refractive error ranging between +4.0 and +7.0 diopters. The control group included 30 females and 16 males aged 15-46 (mean 17.5 \pm 4.5 ) years with a refractive error ranging between +4.0 and +6.0 diopters.

\section{Improvement in visual acuity in study group}

At the baseline examination, mean visual acuity was $0.42 \log$ MAR; end-of-treatment examination revealed that the mean visual acuity had improved to $0.16 \log$ MAR, demonstrating a mean improvement of $2.6 \log$ MAR lines. This improvement was statistically significant $(z=-3.24, P=0.001)$. BCVA in the amblyopic eyes increased by an average of $50.8 \%$. The breakdown of BCVA improvement was as follows: $37.7 \%$ improved between 3.3 and 6.6 lines, 39.6\% improved between 2.0 and 2.9 lines, $20.8 \%$ improved between 1.0 and 1.8 lines, and one individual had no improvement or regression of BCVA in the amblyopic eye. Total improvement of 
BCVA was not influenced by initial BCVA. Figure 3 shows the logMAR BCVA from baseline to the end-of-treatment examination.

\section{Improvement in visual acuity in control group}

At the baseline examination, the mean visual acuity was $0.4 \log$ MAR; end-of-treatment examination showed that mean visual acuity had improved to $0.32 \log$ MAR, demonstrating a mean improvement of $0.08 \log$ MAR lines. This improvement was not statistically significant $(z=-10.2$, $P=0.07)$.

\section{Improvement in contrast sensitivity in the study group}

Mean improvements of $75.5 \%, 68.7 \%, 52.2 \%, 42.2 \%$, and $27.5 \%$ were found in the five CSF levels respectively, ie, A (1.5), B (3), C (6), D (12), and E (18) from baseline to treatment end. The five CSF levels were analyzed across the four time points, ie, baseline, visit 1, visit 2, and treatment end, using repeated-measures analysis of variance. The two-way interaction between time and CSF was significant $\left(F[12,660]=2.29, P<0.007, \eta^{2}=0.04\right.$, Figure 4$)$. CSF differed significantly at the different time points. However, for most CSF levels, the second visit and treatment end did not significantly differ from each other, but the baseline and first visit data showed difference. In terms of the number of treatments and CSF, the number of treatment sessions were significantly correlated with improvement levels. Improvement of CSF was not influenced by age.

\section{Improvement in contrast sensitivity in study group}

Baseline CSF at 1.5, 3, 6, 12, and 18 cycles per degree spatial frequencies was $8.65,7.38,6.30,5.2$, and 2.15 , respectively.

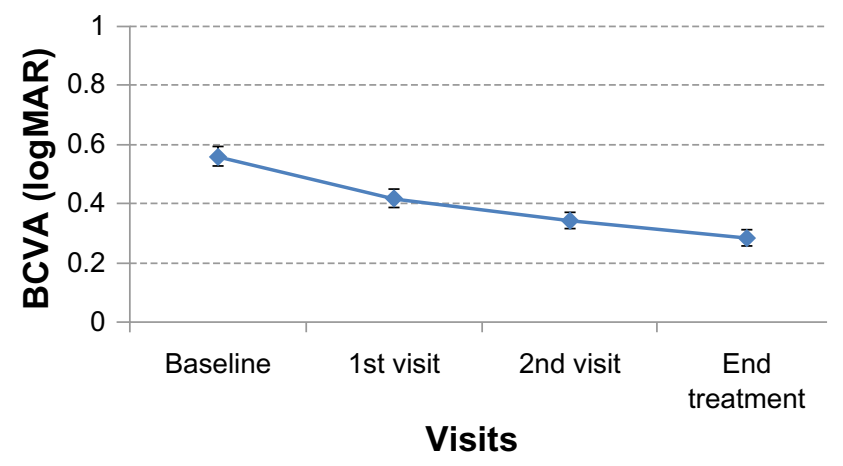

Figure 3 LogMAR best-corrected visual acuity from baseline, first visit, second visit, and treatment end.

Abbreviations: logMAR, logarithm of the minimum angle of resolution; BCVA, best-corrected visual acuity.

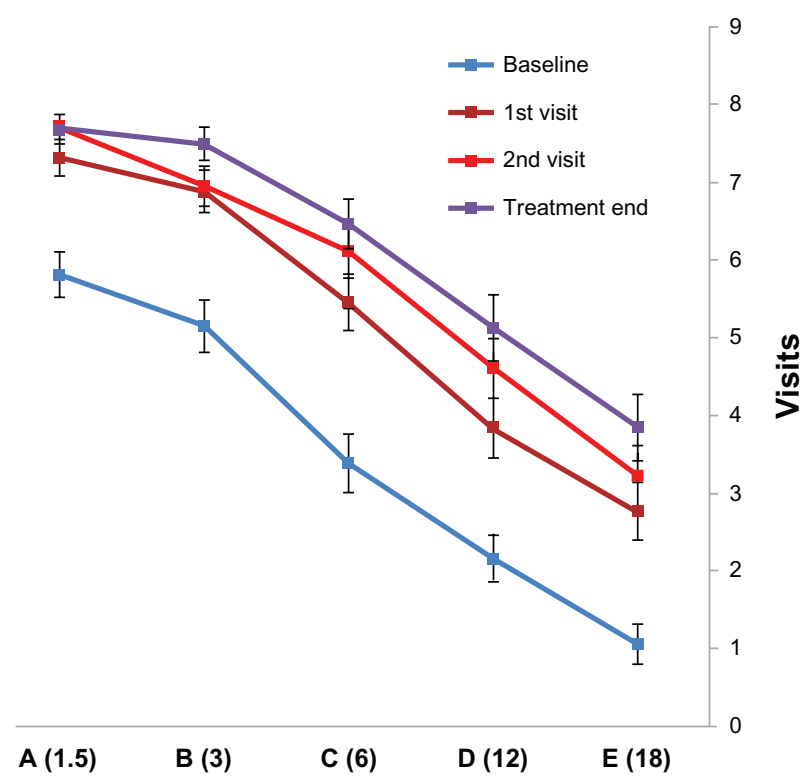

CSF at $1.5,3,6,12$, and 18 cycles per degree

Figure 4 Contrast sensitivity function from baseline, first visit, second visit, and treatment end with standard error bars.

Abbreviation: CSF, contrast sensitivity function.

After placebo training, CSF at 1.5, 3, 6, 12, and 18 cycles per degree was $7.38,7.92,6.4,5.92$, and 3.38 , respectively. The difference was not statistically significant $(z=11.5, P=0.08)$. None of the treated eyes showed a drop in visual acuity. Manifest refractions remained unchanged during the study. All patients were followed up to 4 months. By 4 months, five subjects in the study group had dropped out, but the others were followed up to 8 months.

\section{Improvement in stereoacuity}

Mean stereoacuity was 250 (range 250-400) seconds of arc in the study group. Only five patients in the study group showed an improvement to $20-100$ seconds of arc. The others showed no improvement. Stereoacuity did not differ in the control group, which had a mean stereoacuity of 300 (range 250-400) seconds of arc.

\section{Discussion}

The term "perceptual learning" describes a process whereby practicing certain visual tasks leads to an improvement in visual performance. Visual performance can be improved with repetitive practice on specific controlled visual tasks. These repetitive tasks initiate neural modifications that can lead to improvement in neuronal efficiency. Such neural modifications indicate the presence of brain plasticity. Brain plasticity in visual function of adults has been shown in various studies. ${ }^{19-21}$ This technology has been demonstrated 
clinically in the treatment of adult amblyopia. ${ }^{14,16-19}$ Polat et al studied 54 adult amblyopic patients who were randomized to a perceptual vision treatment program or a placebo vision training program for amblyopia. Pretreatment visual acuity in both study groups was $0.42 \log$ MAR, and this improved by 2.5 lines in the perceptual vision treatment group, with no improvement in the control group. CSF improved to within the normal range. These improvements in acuity and CSF were sustained after 12 months. ${ }^{14}$ Another recent study has demonstrated the efficacy of this technique in amblyopic children aged 7-8 years who had failed conventional patching. ${ }^{15}$ Other studies have demonstrated the efficacy of perceptual vision therapy in improving unaided vision in low myopic patients ${ }^{20}$ and improving unaided vision in presbyopic patients, ${ }^{21}$ as well as in post-refractive surgery patients. ${ }^{22}$

The results of our study demonstrate the efficacy of perceptual vision therapy in improving visual acuity. The improvement of 2.6 logMAR lines in visual acuity is encouraging and is consistent with the results of previous studies. ${ }^{14,15,20}$ While the control group did demonstrate a minimal positive trend, no significant changes in vision were recorded. This suggests that the improvement in the treatment group was not a result of memorizing the vision charts used. CSF also improved after perceptual vision therapy. Although we have good results with this treatment, further studies of this technique and longer follow-up are needed to evaluate the efficacy of this treatment.

\section{Disclosure}

The authors report no conflicts of interest in this work.

\section{References}

1. Rasch E, Swift H, Riesen AH, Chow KL. Altered structure and composition of retinal cells in dark-reared mammals. Exp Cell Res. 1961;25: 348-363.

2. Wendell-Smith CP. Effect of light deprivation on the postnatal development of the optic nerve. Nature. 1964;204:707.

3. Chow KL. Failure to demonstrate changes in the visual system of monkeys kept in darkness or colored light. J Comp Neurol. 1955;102: 597-606.
4. Chauban S, Marshall J. The interpretation of optical coherence tomography image of the retina. Invest Ophthalmol Vis Sci. 1999;40: 2332-2342.

5. Webber JL, Wood J. Amblyopia: prevalence, natural history, functional effects and treatment. Clin Exp Optom. 2005;88:365-375.

6. Headon MP, Powell TPS. Cellular changes in the lateral geniculate nucleus of infant monkeys after suture of the eyelids. J Anat. 1973;116: 135-145.

7. Sherman SM, Wilson JR. Behavioral and morphological evidence for binocular competition in the postnatal development of the dog's visual system. J Comp Neurol. 1975;161:183-195.

8. Von Noorden GK. Histological studies of the visual system in monkeys with experimental amblyopia. Invest Ophthalmol Vis Sci. 1973;12: 727-738.

9. Wiesel TN, Hubel DH. Effect of visual deprivation on morphology and physiology of cells in the cat's lateral geniculate body. J Neurophysiol. 1963;26:978-993.

10. Von Noorden GK, Crawford MLJ, Levacy RA. The lateral geniculate nucleus in human anisometropic amblyopia. Invest Ophthalmol Vis Sci. 1983;24:788-790.

11. Von Noorden GK, Crawford ML. The lateral geniculate nucleus in human strabismic amblyopia. Invest Ophthalmol Vis Sci. 1992;33: 2729-2732.

12. Levi DM, Polat U. Neural plasticity in adults with amblyopia. Proc Natl Acad Sci U S A. 1996;93:6830-6834.

13. Levi DM, Polat U, Hu YS. Improvement in vernier acuity in adults with amblyopia. Practice makes better. Invest Ophthalmol Vis Sci. 1997;38: 1493-1510.

14. Polat U, Ma-Naim T, Belkin M, Sagi D. Improving vision in adult amblyopia by perceptual learning. Proc Natl Acad Sci USA. 2004;101: 6692-6697.

15. Polat U, Ma-Naim T, Spierer A. Treatment of children with amblyopia by perceptual learning. Vision Res. 2009;49:2599-2603.

16. Polat U, Sagi D. Spatial interactions in human vision: from near to far via experience-dependent cascades of connections. Proc Natl Acad Sci US A. 1994;91:1206-1209.

17. Levi DM, Polat U. Neural plasticity in adults with amblyopia. Proc Natl Acad Sci U S A. 1996;93:6830-6834.

18. Polat U, Sagi D, Norcia AM. Abnormal long-range spatial interactions in amblyopia. Vision Res. 1997;37:737-744.

19. Polat U, Mizobe K, Pettet MW, Kasamatsu T, Norcia AM. Collinear stimuli regulate visual responses depending on cell's contrast threshold. Nature. 1998;391:580-584.

20. Tan DTH, Fong A. Efficacy of neural vision therapy to enhance contrast sensitivity function and visual acuity in low myopia. J Cataract Refract Surg. 2008;34:570-577.

21. Durrie D, Shaw P. Computer-based primary visual cortex training for the treatment of low myopia and early presbyopia. Trans Am Ophthalmol Soc. 2007;105:132-114.

22. Lim KL, Fam BH. NeuroVision treatment for low myopia following LASIK regression. J Refract Surg. 2006;22:407-408.
Clinical Ophthalmology

\section{Publish your work in this journal}

Clinical Ophthalmology is an international, peer-reviewed journal covering all subspecialties within ophthalmology. Key topics include: Optometry; Visual science; Pharmacology and drug therapy in eye diseases; Basic Sciences; Primary and Secondary eye care; Patient Safety and Quality of Care Improvements. This journal is indexed on Submit your manuscript here: http://www.dovepress.com/clinical-ophthalmology-journal

\section{Dovepress}

PubMed Central and CAS, and is the official journal of The Society of Clinical Ophthalmology (SCO). The manuscript management system is completely online and includes a very quick and fair peer-review system, which is all easy to use. Visit http://www.dovepress.com/ testimonials.php to read real quotes from published authors. 